\title{
MAXIMAL DOUGLAS SUBALGEBRAS AND MINIMAL SUPPORT POINTS
}

\author{
CARROLL GUILLORY AND KEIJI IZUCHI
}

(Communicated by Paul S. Muhly)

\begin{abstract}
Let $B$ denote a Douglas algebra. Then $B$ has a maximal Douglas subalgebra if and only if the set of points outside the maximal ideal space of $B$ has a minimal support point.
\end{abstract}

\section{INTRODUCTION}

Let $H^{\infty}$ be the space of bounded analytic functions on the open unit disc $D$, and let $L^{\infty}$ be the space of bounded measurable functions on the unit circle $\partial D$ with respect to the Lebesgue measure $d \theta / 2 \pi$. Identifying $H^{\infty}$ with these boundary functions, we may consider that $H^{\infty}$ is an essentially sup-norm closed subalgebra of $L^{\infty}$. An essentially sup-norm closed subalgebra $B$ of $L^{\infty}$ containing $H^{\infty}$ is called a Douglas algebra. We denote by $C$ the space of continuous functions on $\partial D$. Then it is well known that $H^{\infty}+C$ is a Douglas algebra and contains $H^{\infty}$ as a maximal Douglas subalgebra [10]. Also in [4], Guillory, Izuchi, and Sarason showed that there is a Douglas algebra $B$ such that $H^{\infty}+C \subsetneq B \subsetneq L^{\infty}$ and $B$ has a maximal Douglas subalgebra. On the other hand, Sundberg [11] proved that $L^{\infty}$ does not have a maximal Douglas subalgebra. In this paper, we answer to a question when a given Douglas algebra has a maximal Douglas subalgebra.

For a Douglas algebra $B$, we let $M(B)$ denote the maximal ideal space of $B$. A Gelfand transform of $f$ in $B$ is denoted by the same symbol $f . M(B)$ may be identified with a closed subset of $M\left(H^{\infty}\right)$. Then $M\left(L^{\infty}\right)$ becomes the Shilov boundary for $B$ and $M\left(H^{\infty}+C\right)=M\left(H^{\infty}\right) \backslash D$. For a point $x$ in $M\left(H^{\infty}\right)$, we denote by $\mu_{x}$ its representing measure on $M\left(L^{\infty}\right)$, that is, $f(x)=\int_{M\left(L^{\infty}\right)} f d \mu_{x}$ for every $f$ in $H^{\infty}$. We put

$$
H_{\text {supp } \mu_{x}}^{\infty}=\left\{f \in L^{\infty} ; f_{\mid \operatorname{supp} \mu_{x}} \in H_{\mid \operatorname{supp} \mu_{x}}^{\infty}\right\} ;
$$

then $H_{\text {supp } \mu_{x}}^{\infty}$ is a Douglas algebra and

$$
M\left(H_{\text {supp } \mu_{x}}^{\infty}\right)=M\left(L^{\infty}\right) \cup\left\{\zeta \in M\left(H^{\infty}\right) ; \operatorname{supp} \mu_{\zeta} \subset \operatorname{supp} \mu_{x}\right\}
$$

Received by the editors December 4, 1989 and, in revised form, March 23, 1991.

1991 Mathematics Subject Classification. Primary 46J15, 46J30.

The second author was supported in part by Grant-in-Aid for General Scientific Research (No. 01540161), The Ministry of Education, Science, and Culture. 
For a subset $\Omega$ of $M\left(H^{\infty}\right)$, a point $x$ in $\Omega$ is called a minimal support point for $\Omega$ if there are no points $y$ in $\Omega$ such that supp $\mu_{y} \subsetneq \operatorname{supp} \mu_{x}$. The following is our main theorem.

Theorem 1. Let $B$ be a Douglas algebra. Then $B$ has a maximal Douglas subalgebra if and only if $M\left(H^{\infty}\right) \backslash M(B)$ has a minimal support point. If $x$ is a minimal support point for $M\left(H^{\infty}\right) \backslash M(B)$, then $B \cap H_{\text {supp } \mu_{x}}^{\infty}$ is a maximal Douglas subalgebra of $B$, and every maximal Douglas subalgebra is given by this form.

In $\S 2$, we prove Theorem 1 . In $\S 3$, we give some applications of Theorem 1 .

\section{Proof OF TheOREM 1}

A function $\psi$ in $H^{\infty}$ with $|\psi|=1$ on $M\left(L^{\infty}\right)$ is called inner. The famous Chang-Marshall theorem $[1,9]$ says that if $B$ is a Douglas algebra then $B$ is generated by $H^{\infty}$ and complex conjugates of inner functions $\psi$ with $\bar{\psi} \in B$ and $M(B)$ is the set of points $x$ in $M\left(H^{\infty}\right)$ such that $|\psi(x)|=1$ for every inner function $\psi$ with $\bar{\psi} \in B$, that is, $M(B)=\left\{x \in M\left(H^{\infty}\right) ; B_{\mid \operatorname{supp} \mu_{x}}=\right.$ $\left.H_{\mid \text {supp } \mu_{x}}^{\infty}\right\}$. We use these facts frequently. To prove our theorem, we need the following unpublished result of Sarason (see [3, Proposition 3.4]).

Lemma 1. Let $B_{1}$ and $B_{2}$ be Douglas algebras. Then $M\left(B_{1} \cap B_{2}\right)=M\left(B_{1}\right) \cup$ $M\left(B_{2}\right)$.

Proof of Theorem 1. Let $A$ be a maximal Douglas subalgebra of $B$. Since $M(B) \subsetneq M(A)$, take a point $x$ in $M(A) \backslash M(B)$. To prove that $x$ is a minimal support point for $M\left(H^{\infty}\right) \backslash M(B)$, suppose not. Then there exists $y$ in $M\left(H^{\infty}\right) \backslash M(B)$ such that $\operatorname{supp} \mu_{y} \subsetneq \operatorname{supp} \mu_{x}$. Let $C=B \cap H_{\text {supp } \mu_{y}}^{\infty}$. Since $x \in M(A)$, it follows that $A_{\mid \operatorname{supp} \mu_{x}}=H_{\mid \operatorname{supp} \mu_{x}}^{\infty}$, so that $A_{\mid \operatorname{supp} \mu_{y}}=H_{\mid \text {supp } \mu_{y}}^{\infty}$. Therefore we have $A \subset C \subset B$. By Lemma 1,

$$
M(C)=M(B) \cup\left\{\zeta \in M\left(H^{\infty}\right) ; \operatorname{supp} \mu_{\zeta} \subset \operatorname{supp} \mu_{y}\right\} .
$$

Since $y \in M(C)$ and $y \notin M(B)$, it follows that $M(B) \subsetneq M(C)$ and $C \subsetneq B$. Since $x \notin M(C)$ and $x \in M(A)$, it follows that $M(C) \subsetneq M(A)$ and $A \subsetneq C$. Hence $A \subsetneq C \subsetneq B$, contradicting the maximality of $A$.

Since $A \subset H_{\text {supp } \mu_{x}}^{\infty}$, we have $A \subset B \cap H_{\text {supp } \mu_{x}}^{\infty}$. Since $x \notin M(B)$ and $x \in M\left(B \cap H_{\text {supp } \mu_{x}}^{\infty}\right)$, it follows that $B \cap H_{\text {supp } \mu_{x}}^{\infty} \subsetneq B$. By the maximality of $A$, we get $A=B \cap H_{\text {supp } \mu_{x}}^{\infty}$.

To show the converse, let $x$ be a minimal support point for $M\left(H^{\infty}\right) \backslash M(B)$ and let $A=B \cap H_{\text {supp } \mu_{x}}^{\infty}$. Then $A \subset B$ and $M(A)=M(B) \cup\left\{\zeta \in M\left(H^{\infty}\right)\right.$; $\left.\operatorname{supp} \mu_{\zeta} \subset \operatorname{supp} \mu_{x}\right\}$. Since $x \in M(A)$ and $x \notin M(B)$, we have $A \subsetneq B$. Since $x$ is a minimal support point for $M\left(H^{\infty}\right) \backslash M(B)$, if $\operatorname{supp} \mu_{\zeta} \subsetneq \operatorname{supp} \mu_{x}$ then $\zeta \in M(B)$. Hence

$$
M(A)=M(B) \cup\left\{\zeta \in M\left(H^{\infty}\right) ; \operatorname{supp} \mu_{\zeta}=\operatorname{supp} \mu_{x}\right\}
$$

Let $Y$ be a Douglas algebra such that $A \subsetneq Y \subset B$. By Chang-Marshall's theorem, there is an inner function $\psi$ such that $\bar{\psi} \notin A$ and $\bar{\psi} \in Y \subset B$. Then $|\psi|=1$ on $M(B)$ and $|\psi(x)|<1$. Hence $|\psi|<1$ on $M(A) \backslash M(B)$. Since $M(B) \subset M(Y) \subset M(A)$, it follows that $M(B)=M(Y)$. Consequently $B=Y$ and this implies that $A$ is a maximal Douglas subalgebra of $B$. 
Remark. By the above proof, if $A \subsetneq B$ then $A$ is a maximal Douglas subalgebra of $B$ if and only if supp $\mu_{x}=\operatorname{supp} \mu_{y}$ for every $x, y$ in $M(A) \backslash M(B)$.

\section{Applications}

A Blaschke product $b$ with zeros $\left\{z_{n}\right\}_{n}$ in $D$ is called sparse if

$$
\lim _{k \rightarrow \infty} \prod_{n: n \neq k}\left|\frac{z_{k}-z_{n}}{1-\bar{z}_{n} z_{k}}\right|=1 \text {. }
$$

The above condition implies that $\left\{z_{n}\right\}_{n}$ is interpolating for $H^{\infty}$, that is, for every bounded sequence $\left\{a_{n}\right\}_{n}$ there exists $f$ in $H^{\infty}$ such that $f\left(z_{n}\right)=a_{n}$ for every $n$. For every sequence $\left\{y_{n}\right\}_{n}$ in $D$ with $\left|y_{n}\right| \rightarrow 1$, we can find a subsequence that satisfies the sparseness condition. For a function $f$ in $H^{\infty}$, we put $Z(f)=\left\{x \in M\left(H^{\infty}+C\right) ; f(x)=0\right\}$. For a subset $F$ of $L^{\infty}$, we denote by $[F]$ the closed subalgebra of $L^{\infty}$ generated by $F$.

Theorem 2. Let $\psi$ be an inner function such that $\psi$ is not continuous on $\partial D$. Then $\left[H^{\infty}, \bar{\psi}\right]$ has uncountably many maximal Douglas subalgebras.

Proof. We have $M\left(\left[H^{\infty}, \bar{\psi}\right]\right)=\left\{\zeta \in M\left(H^{\infty}+C\right) ;|\psi(\zeta)|=1\right\}$. For $\zeta \in$ $M\left(H^{\infty}\right) \backslash M\left(\left[H^{\infty}, \bar{\psi}\right]\right)$, there is a point $x$ in $Z(\psi)$ such that $\operatorname{supp} \mu_{x} \subset \operatorname{supp} \mu_{\zeta}$. Hence to study minimal support points for $M\left(H^{\infty}\right) \backslash M\left(\left[H^{\infty}, \bar{\psi}\right]\right)$ is the same as to study minimal support points for $Z(\psi)$, so we concentrate on $Z(\psi)$.

For $x, y$ in $Z(\psi)$, we define the order as follows; $y \leq x$ if supp $\mu_{y} \subset$ $\operatorname{supp} \mu_{x}$, and $x=y$ if $\operatorname{supp} \mu_{y}=\operatorname{supp} \mu_{x}$. By Hoffman's unpublished result [7], if $\operatorname{supp} \mu_{y} \cap \operatorname{supp} \mu_{x} \neq \varnothing$ then $\operatorname{supp} \mu_{y} \subset \operatorname{supp} \mu_{x}$ or $\operatorname{supp} \mu_{y} \supset \operatorname{supp} \mu_{x}$. Hence $Z(\psi)$ becomes an ordered set. Let $\left\{x_{\alpha}\right\}_{\alpha \in \Lambda}$ be a totally ordered subset of $Z(\psi)$. We denote by $F_{\alpha}$ the closure of $\left\{x_{\beta} ; \beta \in \Lambda, \beta \leq \alpha\right\}$ in $M\left(H^{\infty}\right)$. Then $\left\{F_{\alpha}\right\}_{\alpha}$ is a family of compact decreasing subsets of $Z(\psi)$. Therefore, there is a point $x_{0}$ in $\bigcap_{\alpha} F_{\alpha}$, and we have supp $\mu_{x_{0}} \subset \operatorname{supp} \mu_{x_{\alpha}}$ and $x_{0} \leq x_{\alpha}$. By Zorn's lemma, $Z(\psi)$ has a minimal element; hence $M\left(H^{\infty}\right) \backslash M\left(\left[H^{\infty}, \bar{\psi}\right]\right)$ has a minimal support point. By Theorem $1,\left[H^{\infty}, \bar{\psi}\right]$ has a maximal Douglas subalgebra.

Let $b$ be a sparse Blaschke product with zeros $\left\{z_{n}\right\}_{n}$ such that $\left|\psi\left(z_{n}\right)\right| \rightarrow 0$ $(n \rightarrow \infty)$. By [5, p. 205], $Z(b)$ is contained in the closure of $\left\{z_{n}\right\}_{n}$ in $M\left(H^{\infty}\right)$. Then $Z(b) \subset Z(\psi)$. By the second paragraph, for each $\zeta \in Z(b)$ there is a minimal element $\zeta_{0}$ in $Z(\psi)$ such that $\zeta_{0} \leq \zeta$. By Theorem $1,\left[H^{\infty}, \bar{\psi}\right]$ has maximal Douglas sut.algebras $\left\{\left[H^{\infty}, \bar{\psi}\right] \cap H_{\text {supp } \mu_{\zeta_{0}}}^{\infty} \zeta \in Z(b)\right\}$. By [4, p. 5], $\operatorname{supp} \mu_{\zeta} \cap \operatorname{supp} \mu_{\lambda}=\varnothing$ for distinct $\zeta, \lambda \in Z(b)$. Since $\operatorname{supp} \mu_{\zeta_{0}} \subset \operatorname{supp} \mu_{\zeta}$, it follows that $\left[H^{\infty}, \bar{\psi}\right] \cap H_{\text {supp } \mu_{\zeta_{0}}}^{\infty} \neq\left[H^{\infty}, \bar{\psi}\right] \cap H_{\text {supp } \mu \lambda_{0}}^{\infty}$ for distinct $\zeta, \lambda \in Z(b)$. Since $Z(b)$ is homeomorphic to $\beta N \backslash N[5$, p. 205] where $\beta N$ is the StoneČech compactification of integers $N$, we get our assertion.

Theorem 3. Let $B$ be a Douglas algebra with $B \subsetneq L^{\infty}$. Let $\psi$ be an inner function with $\bar{\psi} \notin B$. Then $[B, \bar{\psi}]$ has a maximal Douglas subalgebra.

Proof. Let $x \in M(B) \backslash M([B, \bar{\psi}])$. Then $|\psi(x)|<1$. By the proof of Theorem 2, there is a minimal support point $y$ for $M\left(H^{\infty}\right) \backslash M\left(\left[H^{\infty}, \bar{\psi}\right]\right)$ such that $\operatorname{supp} \mu_{y} \subset \operatorname{supp} \mu_{x}$. To prove that $y$ is a minimal support point for $M\left(H^{\infty}\right) \backslash M([B, \bar{\psi}])$, suppose that there is a point $\zeta$ in $M\left(H^{\infty}\right) \backslash M([B, \bar{\psi}])$ such that $\operatorname{supp} \mu_{\zeta} \subsetneq \operatorname{supp} \mu_{y}$. Since $x \in M(B)$, we have $y \in M(B)$, so that 
$\zeta \in M(B)$. Since $\zeta \notin M([B, \bar{\psi}]),|\psi(\zeta)|<1$. This contradicts the minimality of $y$ for $M\left(H^{\infty}\right) \backslash M\left(\left[H^{\infty}, \bar{\psi}\right]\right)$. Hence $y$ is a minimal support point. By Theorem 1, we get Theorem 3.

The following theorem shows that there exists a Douglas algebra that does not have any maximal Douglas subalgebras.

Theorem 4. Let $\left\{\psi_{n}\right\}_{n=1}^{\infty}$ be a sequence of inner functions such that $\psi_{n+1}=0$ on $\left\{\zeta \in M\left(H^{\infty}+C\right) ;\left|\psi_{n}(\zeta)\right|<1\right\}$ for every $n$. Then $\left[H^{\infty}, \bar{\psi}_{n} ; n=1,2, \ldots\right]$ does not have a maximal Douglas subalgebra.

Proof. Put $B=\left[H^{\infty}, \bar{\psi}_{n} ; n=1,2, \ldots\right]$. Let $x \in M\left(H^{\infty}\right) \backslash M(B)$. We shall prove that there is a point $y$ in $M\left(H^{\infty}\right) \backslash M(B)$ such that supp $\mu_{y} \subsetneq$ supp $\mu_{x}$. Since $x \notin M(B)$, it follows that $\left|\psi_{n}(x)\right|<1$ for some $n$; hence $\left\{\zeta \in M\left(H_{\text {supp } \mu_{x}}^{\infty}\right) ;\left|\psi_{n}(\zeta)\right|<1\right\}$ is a nonvoid open subset of $M\left(H_{\text {supp } \mu_{x}}^{\infty}\right)$. But it is not closed in $M\left(H_{\text {supp } \mu_{x}}^{\infty}\right)$. For if it is closed in $M\left(H_{\text {supp } \mu_{x}}^{\infty}\right)$, by the Shilov idempotent theorem, there is a function $g$ in $H_{\text {supp } \mu_{x}}^{\infty}$ such that $g^{2}=g$ and $\left\{\zeta \in M\left(H_{\text {supp } \mu_{x}}^{\infty}\right) ; g(\zeta)=1\right\}=\left\{\zeta \in M\left(H_{\text {supp } \mu_{x}}^{\infty}\right) ;\left|\psi_{n}(\zeta)\right|=1\right\}$. Since $g=1$ on $M\left(L^{\infty}\right)$ and $\left|\psi_{n}(x)\right| \neq 1$, we have $0=g(x)=\int_{M\left(L^{\infty}\right)} g d \mu_{x}=1$; this is impossible.

Take a point $y$ in the closure of $\left\{\zeta \in M\left(H_{\operatorname{supp} \mu_{x}}^{\infty}\right) ; \mid \psi_{n}(\zeta)<1\right\}$ such that $\left|\psi_{n}(y)\right|=1$. Since $\operatorname{supp} \mu_{\zeta} \subset \operatorname{supp} \mu_{x}$ for $\zeta$ in $M\left(H_{\text {supp } \mu_{x}}^{\infty}\right)$ with $\left|\psi_{n}(\zeta)\right|<$ 1 , we have $\operatorname{supp} \mu_{y} \subset \operatorname{supp} \mu_{x}$. Since $\psi_{n}$ is constant on supp $\mu_{y}$ and $\psi_{n}$ is not constant on $\operatorname{supp} \mu_{x}$, we have $\operatorname{supp} \mu_{y} \subsetneq \operatorname{supp} \mu_{x}$. Since $\psi_{n+1}=0$ on $\left\{\zeta \in M\left(H^{\infty}+C\right) ;\left|\psi_{n}(\zeta)\right|<1\right\}$, we have $\psi_{n+1}(y)=0$, so that $y \notin$ $M(B)$. Hence every point in $M\left(H^{\infty}\right) \backslash M(B)$ is not a minimal support point for $M\left(H^{\infty}\right) \backslash M(B)$. By Theorem 1, we get Theorem 4 .

Corollary 1. There is a function $f$ in $L^{\infty}$ such that $\left[H^{\infty}, f\right]$ does not have a maximal Douglas subalgebra.

Proof. By [11] there is a sequence of inner functions $\left\{\psi_{n}\right\}_{n}$ that satisfy the condition of Theorem 4. Take $f$ in $L^{\infty}$ such that $\left[H^{\infty}, f\right]=\left[H^{\infty}, \bar{\psi}_{n}\right.$; $n=1,2, \ldots]$ (see [8, Lemma 2.2]). Then $f$ satisfies our assertion.

For a Douglas algebra $B$, a Douglas algebra $A$ with $B \subsetneq A$ is called a minimal superalgebra of $B$ if there are no Douglas algebras $J$ such that $B \subsetneq$ $J \subsetneq A$; that is, $B$ is a maximal Douglas subalgebra of $A$. If $A$ is a minimal superalgebra of $B$, then $A=[B, \bar{\psi}]$ for some inner function $\psi$. The following theorem is a restatement of Theorem 1 and Remark.

Theorem 5. Let $B$ be a Douglas algebra. Then $B$ has a minimal superalgebra if and only if there is an inner function $\psi, \bar{\psi} \notin B$ such that $\operatorname{supp} \mu_{x}=\operatorname{supp} \mu_{y}$ for every $x, y$ in $\{\zeta \in M(B) ;|\psi(\zeta)|<1\}$. Under the above situation, $[B, \bar{\psi}]$ becomes a minimal superalgebra of $B$.

Corollary 2. For every function $f$ in $L^{\infty}$ with $f \notin H^{\infty},\left[H^{\infty}, f\right]$ does not have any minimal superalgebras.

Proof. Put $B=\left[H^{\infty}, f\right]$. By [8, Lemma 2.2], there is a sequence of inner functions $\left\{\psi_{n}\right\}_{n}$ such that $B=\left[H^{\infty}, \bar{\psi}_{n} ; n=1,2, \ldots\right]$. Then $M(B)$ is a closed $G_{\delta}$-subset of $M\left(H^{\infty}\right)$. Let $g$ be a nonnegative continuous function on $M\left(H^{\infty}\right)$ such that $g=1$ on $M(B)$ and $0 \leq g<1$ on $M\left(H^{\infty}\right) \backslash M(B)$. To prove our assertion, let $\psi$ be an inner function such that $\bar{\psi} \notin B$. Then 
$Z(\psi) \cap M(B) \neq \varnothing$. By the corona theorem (see [2, p. 318]), there is a sequence $\left\{z_{n}\right\}_{n}$ in $D$ such that $g\left(z_{n}\right) \rightarrow 1$ and $\psi\left(z_{n}\right) \rightarrow 0$. Let $b$ be a Blaschke product with zeros $\left\{z_{n}\right\}_{n}$. We may assume that $b$ is sparse. Then we have $Z(b) \subset M(B) \cap Z(\psi)$ and $\operatorname{supp} \mu_{x} \neq \operatorname{supp} \mu_{y}$ for $x, y$ in $Z(b), x \neq y$. By Theorem 5 , we get our assertion.

\section{ACKNOWLEDGMENT}

The authors would like to thank the referee for shortening the proof of Theorem 1 .

\section{REFERENCES}

1. S.-Y. Chang, A characterization of Douglas subalgebras, Acta Math. 137 (1976), 81-89.

2. J. Garnett, Bounded analytic functions, Academic Press, New York and London, 1981.

3. P. Gorkin, Decomposition of the maximal ideal space of $L^{\infty}$, Thesis, Michigan State Univ., East Lansing, MI, 1982.

4. C. Guillory, K. Izuchi, and D. Sarason, Interpolating Blaschke products and division in Douglas algebras, Proc. Roy. Irish Acad. Sect. A 84 (1984), 1-7.

5. K. Hoffman, Banach spaces of analytic functions, Prentice-Hall, Englewood Cliffs, NJ, 1962.

6. __ Bounded analytic functions and Gleason parts, Ann. of Math. (2) 86 (1967), 74-111.

7. __ Unpublished note.

8. K. Izuchi, Countably generated Douglas algebras, Trans. Amer. Math. Soc. 299 (1987), 171-192.

9. D. Marshall, Subalgebras of $L^{\infty}$ containing $H^{\infty}$, Acta Math. 137 (1976), 91-98.

10. D. Sarason, Function theory on the unit circle, Virginia Polytechnic Inst. and State Univ., Blacksburg, VA, 1978.

11. C. Sundberg, A note on algebras between $L^{\infty}$ and $H^{\infty}$, Rocky Mountain J. Math. 11 (1981), 333-336.

Department of Mathematics, University of Southwestern louisiana, Lafayette, LOUISIANA 70504

Department of Mathematics, Kanagawa University, Yokohama 221, Japan 